\title{
High-energy constraints on the direct detection of MSSM neutralinos
}

\author{
John Ellis \\ TH Division, CERN, Geneva, Switzerland
}

\author{
Keith A. Olive, Yudi Santoso,* and Vassilis C. Spanos \\ William I. Fine Theoretical Physics Institute, University of Minnesota, Minneapolis, Minnesota 55455, USA
}

(Received 14 August 2003; published 27 January 2004)

\begin{abstract}
The requirement that the minimal supersymmetric standard model (MSSM) remain an acceptable effective field theory up to energies beyond the weak scale constrains the sparticle spectrum, and hence the permissible ranges of cold dark matter neutralino-proton cross sections. Specifically, squarks are generally much heavier than sleptons if no tachyons are to appear before the grand unified theory (GUT) scale $\sim 10^{16} \mathrm{GeV}$, or even before $10 \mathrm{TeV}$. We display explicitly the allowed ranges of effective squark and slepton masses at the weak scale, and the cross-section ranges allowed if the MSSM is to remain valid without tachyons up to $10 \mathrm{TeV}$ or the GUT scale. The allowed areas in the cross-section-mass plane for both spin-independent and spindependent scattering are significantly smaller than would be allowed if the MSSM were required to be valid only around the weak scale. In addition to a reduction in the maximum cross section, the upper limit on the neutralino mass is greatly reduced when tachyons are avoided, particularly for smaller values of the squark masses.
\end{abstract}

DOI: 10.1103/PhysRevD.69.015005

PACS number(s): 12.60.Jv, 95.35. +d

\section{INTRODUCTION}

At the present time, supersymmetry is a leading candidate for physics beyond the standard model (SM). It provides a technical solution to the problematic naturalness of the hierarchy of mass scales in particle physics [1] and its renormalization-group equations (RGEs) allow the SM parameters to be run up the Planck scale, enabling the gauge couplings to unify at a high-energy grand unified theory (GUT) scale $\sim 10^{16} \mathrm{GeV}$ [2]. Furthermore, with $R$ parity conserved as in the minimal supersymmetric extension of the SM (MSSM), the lightest neutralino $\chi$, which is expected to be the lightest supersymmetric particle (LSP), is a good candidate for providing the cold dark matter required by astrophysics and cosmology [3].

The MSSM contains two Higgs doublets and each SM particle has a superpartner [4]. In order to obtain viable phenomenology, supersymmetry should be broken by soft terms [5]. In the most general case, there are more than 100 new parameters in this soft supersymmetry-breaking sector, suggesting two approaches for doing the MSSM phenomenology. The first approach is to assume that some simplified pattern of supersymmetry breaking is input at the GUT scale, and then evolve the RGEs for the MSSM top-downwards to lower energies, so as to calculate the spectrum at the weak scale. The simplest model of this kind is the CMSSM [6-12], in which the soft supersymmetry-breaking masses $m_{1 / 2}$ of the gauginos and $m_{0}$ of the sfermions, as well as the trilinear parameters $A_{0}$, are each assumed to be universal at the GUT input scale. Extensive studies have been made of this and other GUT input scenarios [13-15].

Another approach is the low-energy effective supersym-

*Electronic address: santoso@physics.umn.edu metric theory (LEEST) approach. In this case, one does not care about any particular mass relation at high-energy scales, and simply uses the weak scale as the input scale [16-18]. The only phenomenological guidance used is provided by the constraints from experimental observations. However, if one allows all the soft supersymmetry-breaking parameters to be free, there will again be too many parameters for a satisfactory phenomenological analysis to be possible, so this approach usually also assumes some simplification at the weak scale, e.g., universal slepton and/or squark masses, in spite of the fact that these universalities would not look natural from the GUT point of view.

One argument for using the LEEST approach is that experiments have not been able to probe energy scales higher than $1 \mathrm{TeV}$, and no one really knows what the higher-energy physics really is. However, loop corrections should not be abandoned, unless we want to throw out quantum field theory altogether, and the RGEs are the best way to treat these. Even if one does not impose any particular structure at some GUT scale, the RGE running from the bottom upwards could in general lead to some sfermions becoming tachyonic at some higher energy scale, i.e., $m_{\tilde{f}}^{2}(Q)<0$ for some $Q$ $>M_{W}$. To avoid this tachyon problem, one must impose suitable positivity constraints on the different sfermion masses in the LEEST. These would depend on the scale up to which the MSSM RGEs are thought to apply, above which one might add some extra fields in a way suitable for avoiding the tachyonic instability. For example, in the CMSSM with nonuniversal Higgs masses (NUHM), it was shown [14] that positivity constraints on the soft Higgs masses at the GUT scale imposes strong constraints on the allowable parameter space.

The CMSSM and LEEST approaches differ in their treatments of this tachyonic problem. If one takes the top-down 
approach of using the GUT scale as the input scale, and makes the CMSSM universality assumption, it is trivial to avoid the tachyon problem by imposing relatively minor restrictions on the MSSM parameters $\mu, A_{0}$, and $\tan \beta$. In a bottom-up LEEST analysis, the tachyon problem is accentuated if one chooses spectra that optimize particular observables. In this paper, we discuss one particular example, that of the neutralino-proton cross section, which is crucial for experiments searching directly for cold dark matter particles via their scattering on ordinary matter. In this case, one may obtain encouragingly large cross sections by choosing relatively light squark masses. However, renormalization effects are prone to driving such light squarks tachyonic at some energy scale that is not far beyond the weak scale. We consider this contrary to one of the primary ambitions of supersymmetry, which was to formulate a plausible extension of the SM that could remain valid all the way up some high energy scale, such as the GUT scale, and make the mass hierarchy natural.

In view of the importance of this issue, we first summarize in Sec. II why we consider that tachyonic sfermions unacceptable. Then, in Sec. III, we exhibit some of the correlations among the observable sparticle masses that arise if we use nontachyonic soft supersymmetry-breaking sfermion masses at the GUT scale as inputs to the MSSM. We emphasize the well-known point, for example, that the effective squark masses at the weak scale must be much greater than the effective slepton masses in this case, even with nonuniversal input scalar masses, unless we start with slepton masses much greater than squark masses at the input scale. This is a general property of any top-down approach, not just the CMSSM, which does not hold in a generic bottom-up approach, such as the LEEST. Then, Sec. IV outlines the more general model we use for our LEEST analysis, in which no hypothesis is made about extrapolability to the GUT scale. Section V compares the elastic scattering cross sections found for different choices of the energy scale up to which the LEEST is postulated to apply without tachyons appearing. Finally, Sec. VI presents our conclusions.

\section{VACUUM INSTABILITY IN THE MSSM}

Let us first consider a sample RGE for the case of a righthanded up-type squark

$$
Q \frac{d m^{2}}{d Q} \simeq \frac{1}{8 \pi^{2}}\left[-\frac{16}{3} g_{3}^{2} M_{3}^{2}-\frac{16}{9} g_{1}^{2} M_{1}^{2}\right]
$$

where $g_{1(3)}$ is the $U(1)_{Y}\left[\mathrm{SU}(3)_{c}\right]$ gauge coupling and $M_{1(3)}$ the corresponding gaugino mass. At the one-loop level, Eq. (1) can be solved exactly using the solutions to the gauge coupling RGEs and the relationship between ratios of gaugino masses and gauge couplings, and yields [19]

$$
\begin{aligned}
m^{2}(Q)= & m^{2}\left(M_{W}\right)-\frac{2}{3 \pi^{2}} g_{3}^{2}\left(M_{3}\right) M_{3}^{2} \ln \left(Q / M_{3}\right) \\
& \times\left\{\frac{1+3 g_{3}^{2}\left(M_{3}\right) \ln \left(Q / M_{3}\right) /\left(16 \pi^{2}\right)}{\left[1+3 g_{3}^{2}\left(M_{3}\right) \ln \left(Q / M_{3}\right) /\left(8 \pi^{2}\right)\right]^{2}}\right\} \\
& -\frac{2}{9 \pi^{2}} g_{1}^{2}\left(M_{1}\right) M_{1}^{2} \ln \left(Q / M_{1}\right) \\
& \times\left\{\frac{1-11 g_{1}^{2}\left(M_{1}\right) \ln \left(Q / M_{1}\right) /\left(16 \pi^{2}\right)}{\left[1-11 g_{1}^{2}\left(M_{1}\right) \ln \left(Q / M_{1}\right) /\left(8 \pi^{2}\right)\right]^{2}}\right\} .
\end{aligned}
$$

From this equation, it is straightforward to see the monotonic decrease in the squark mass at $Q>M_{W}$. In addition, one readily sees that as the gaugino mass (and in particular the gluino mass) increases, the rate at which the squark mass decreases also increases. Therefore, depending on one's choice of $m^{2}\left(M_{W}\right)$, it is quite generic to find that $m^{2}\left(Q_{0}\right)$ $=0$, for some $M_{W}<Q_{0}<M_{\mathrm{GUT}}$.

Problems with tachyonic instabilities were first raised in this context in [19]. The resulting tachyonic behavior of the solution to the RGEs is an indication that the scalars are being expanded about the wrong vacuum. In this case, there are at least two options for treating the problem:

(1) One can introduce new physics at an energy scale below $Q_{0}$, so that the RGE running is affected and the positivity of $m^{2}$ is maintained. Of course, such a solution is by fiat outside the realm of the MSSM and hence of this paper.

(2) If no new physics is introduced below $Q_{0}$, then it must be present at some higher energy $Q>Q_{0}$-perhaps the GUT scale or some intermediate scale-in order to restore the stability of the theory. In this case, however, one expects the squarks to acquire nonzero vacuum expectation values $v(Q) \simeq Q$, related to the new very highenergy scale $Q>Q_{0}$. The severity of such instabilities and the existence of new vacua in the presence of flat directions in the effective potential was particularly stressed in [19], and explicit solutions with $v(Q) \simeq Q$ were exhibited.

To see that the broken vacuum persists down to low energies, consider the evolution of the RGEs at large field values. Any field coupled to the squark with the large vacuum expectation value becomes massive, and hence is unavailable for the radiative running of $m^{2}$ back to positive values. In this case, the solution to the RGEs will have $m^{2}(Q)<0$ for all $Q$ and the theory will possess a low-energy global minimum with charge and color breaking (CCB).

It was argued in $[21,22]$ that this situation could be tolerated, because the Universe would naturally fall into our false vacuum as the cosmological temperature fell, and then take much longer than the present age of the Universe to tunnel into the true vacuum. We find this point of view disturbingly

${ }^{1}$ For discussions concerning CCB vacua in the CMSSM, see [20]. 
anthropic. Moreover, it has been observed that, if the early Universe went through an inflationary phase, it is likely to have fallen directly into the true vacuum, so the lifetime of the false vacuum is irrelevant [23].

Following this debate, cosmological data $\left(\Omega_{\text {tot }} \simeq 1\right.$, nearscale-invariant adiabatic density fluctuations that appear Gaussian, etc. [24]) have come increasingly to favor some variant of inflationary cosmology. At best, the stability conditions on the MSSM might depend on the details of the inflationary cosmology chosen. At worst, their neglect lead to an entirely inconsistent theory. Overall, we think that tolerating tachyonic sfermions would court disaster. Furthermore, since the tachyonic Higgs instability induced by the top Yukawa coupling, leading to dynamical electroweak symmetry breaking, is such an attractive feature of the MSSM, we are reluctant to introduce ambiguity into the mechanism by accepting other tachyonic instabilities in the MSSM.

\section{GUT CORRELATIONS AMONG MSSM PARAMETERS}

The simplest way to avoid the tachyonic instabilities discussed above is to input acceptable parameters at the highenergy scale, as is done in the CMSSM. Assuming input soft supersymmetry-breaking parameters at the GUT or some other high-energy scale, one may use the MSSM RGEs to calculate the sparticle mass spectrum at the weak scale. The top-down RGE evolution leads in general to correlations among the various supersymmetric parameters. Moreover, in some cases, a wide range of these input parameters at the GUT scale corresponds to a specific range down at the weak scale, as we demonstrate in this section.

These striking correlations are indicative of the effect of the RGE evolution of the soft supersymmetry-breaking parameters between the GUT and weak scales. Furthermore, as we will see, certain choices of weak scale masses are never realized in such a top-down approach for the very reason that their origin would correspond to a tachyonic set of parameters at a high energy scale. Therefore, in contrast to the LEEST approach, one cannot treat their values at the weak scale as completely independent quantities, if the model under study corresponds to a more fundamental theory at a higher scale.

We assume in our analysis the following ranges of the sparticle mass parameters at the GUT scale:

$$
\begin{gathered}
m_{\tilde{Q}_{0}}=m_{\tilde{U}_{0}}=m_{\tilde{D}_{0}} \leqslant 2 \mathrm{TeV}, \quad m_{\tilde{L}_{0}}=m_{\tilde{E}_{0}} \leqslant 2 \mathrm{TeV}, \\
m_{1 / 2} \leqslant 2 \mathrm{TeV}, \quad m_{1,2} \leqslant 2 \mathrm{TeV}, \\
\left|A_{0}\right| \leqslant 1 \mathrm{TeV}, \quad \tan \beta \leqslant 58,
\end{gathered}
$$

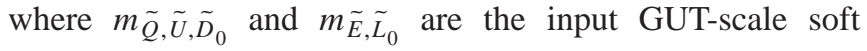
supersymmetry-breaking masses for all three families of squarks and sleptons, respectively, $m_{1 / 2}$ is the common gaugino mass, and $m_{1,2}$ are the soft supersymmetry-breaking masses for the two Higgs doublets. In the CMSSM, one postulates GUT unification relations among the soft supersymmetry-breaking masses of the sfermions and Higgs bosons: $m_{\tilde{Q}_{0}}=m_{\tilde{U}_{0}}=m_{\tilde{D}_{0}}=m_{\tilde{L}_{0}}=m_{\tilde{E}_{0}}=m_{1,2}$. These conditions are relaxed in our analysis here, which is therefore more general than the CMSSM.

To illustrate the weak-scale correlations, we have chosen to present a particular example. We assume universality between the $\mathrm{SU}(3), \mathrm{SU}(2)$, and $\mathrm{U}(1)$ gaugino masses $m_{1 / 2}$ at the GUT scale and discuss the dependences of the weak-scale values of various soft parameters in the sfermion sector. We show in Fig. 1 the weak-scale values of the soft supersymmetry-breaking mass parameters (a) $m_{E_{3}}$ and (b) $m_{\tilde{L}_{3}}$, which appear in the slepton sector of the third generation, and (c) $m_{\tilde{U}_{3}}$ and (d) $m_{\tilde{Q}_{3}}$, which appear in the squark sector of the third generation, as functions of $m_{1 / 2}$. We use a random 50000 -point sample in the range of parameters (3). The different symbols: [light $\times$ signs/dark + signs] correspond to imposing the constraints $m_{\tilde{E}_{0}} \leqslant 500 \mathrm{GeV} /$ between 500 and $1000 \mathrm{GeV}$ for Figs. 1(a) and 1(b), and $m_{\tilde{Q}_{0}}$ $\leqslant 500 \mathrm{GeV} /$ between 500 and $1000 \mathrm{GeV}$ in Figs. 1(c) and $1(\mathrm{~d})$, respectively.

It is immediately apparent that the soft supersymmetrybreaking masses are correlated, especially for large values of the gaugino mass, where the corresponding terms dominate the RGEs for $m_{E_{3}}$ and $m_{L_{3}}$. This can be seen from the approximate one-loop solutions to the RGEs for the sleptons (neglecting the $D$-term contribution for clarity):

$$
\begin{aligned}
& m_{\tilde{E}_{3}}^{2}=m_{\tilde{E}_{30}}^{2}+0.15 m_{1 / 2}^{2}, \\
& m_{\tilde{L}_{3}}^{2}=m_{\tilde{L}_{30}}^{2}+0.52 m_{1 / 2}^{2} .
\end{aligned}
$$

The correlation between the soft supersymmetry-breaking masses $m_{\tilde{Q}_{3}}, m_{\tilde{U}_{3}}$ and the gaugino mass, presented in Figs. $1(\mathrm{c})$ and $1(\mathrm{~d})$, is even tighter, due to the larger value of the combination $g_{3} M_{3}$ relative to either $g_{1} M_{1}$ or $g_{2} M_{2}$, which drives the evolution of the slepton masses and is shown in the following approximate solution:

$$
\begin{aligned}
& m_{\tilde{U}_{3}}^{2}=0.52 m_{\tilde{U}_{30}}^{2}+5.4 m_{1 / 2}^{2}+m_{t}^{2}, \\
& m_{\tilde{Q}_{3}}^{2}=0.04 m_{\tilde{Q}_{30}}^{2}+4.2 m_{1 / 2}^{2}+m_{t}^{2} .
\end{aligned}
$$

In this case, the substantial size of the top Yukawa coupling, which appears in the RGE of $m_{\tilde{Q}_{3}}$, also plays an important role. The boundaries of the allowed regions in Fig. 1 can easily be understood from Eqs. (4) and (5). The upper edge is clearly an artifact of the choice of the upper cutoff on the GUT-scale masses. If one were to select higher ranges for the soft supersymmetry-breaking masses, i.e., greater than 1000 $\mathrm{GeV}$ for the input masses, the correlation region would just move upwards to larger low-energy masses. What is important to note, however, is that the lower right parts of these figures, regions with low mass sfermions and large gaugino masses, are never populated. The lower edge is, in fact, determined by the gaugino mass contribution to the weak-scale masses. Indeed, we see that, because the contributions to the weak-scale masses from the input squark masses are much 

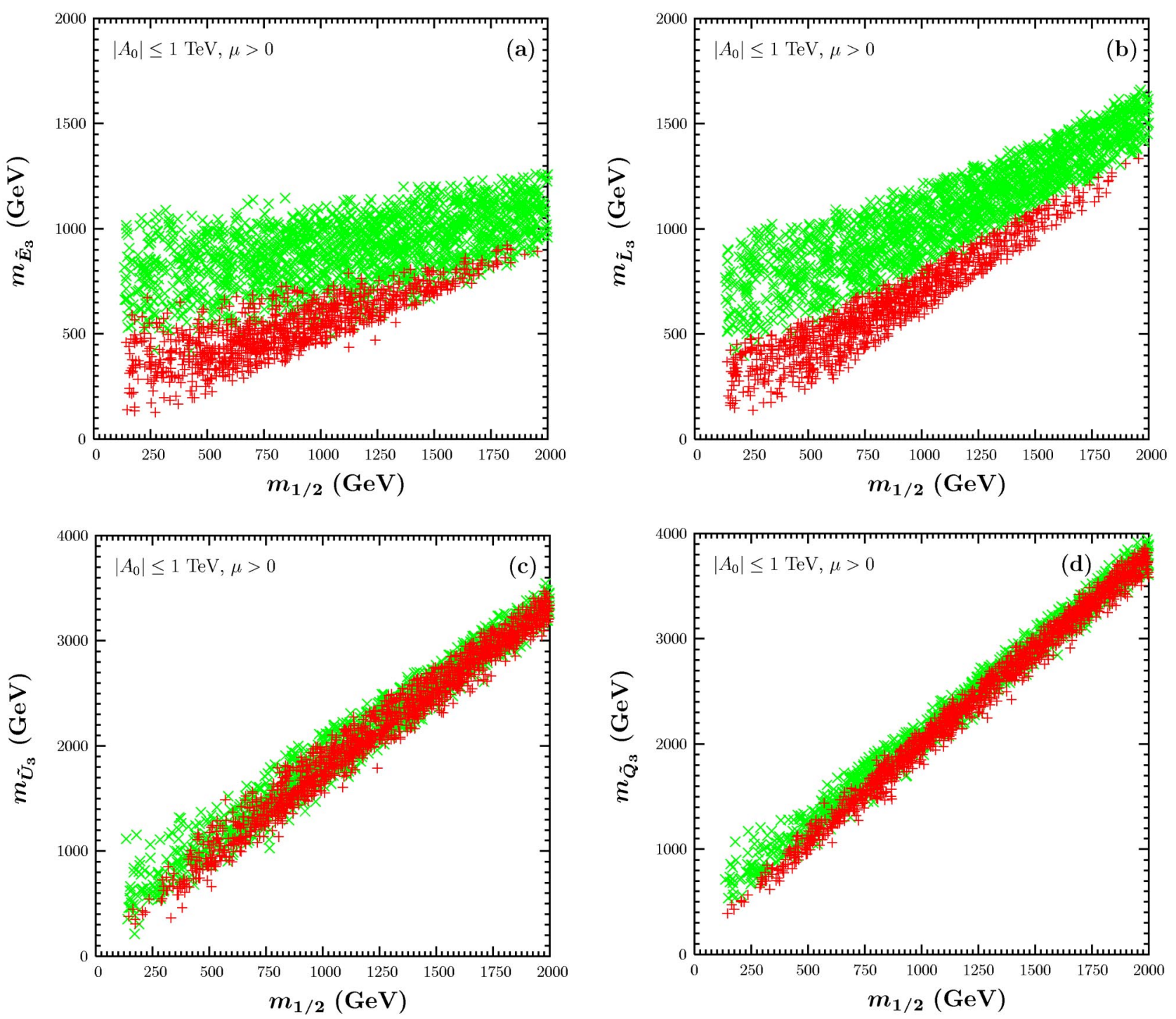

FIG. 1. The weak-scale values of the soft supersymmetry-breaking mass parameters (a) $m_{\tilde{E}_{3}}$, (b) $m_{\tilde{L}_{3}}$, (c) $m_{\tilde{U}_{3}}$, and (d) $m_{\tilde{Q}_{3}}$, as functions of the universal gaugino mass $m_{1 / 2}$ input at the GUT scale. We use light $\times$ signs/dark + signs to denote points with $m_{E_{0}}$ or $m_{\tilde{Q}_{0}} \leqslant 500 \mathrm{GeV} /$ between 500 and $1000 \mathrm{GeV}$ input at the GUT scale.

weaker than those from the input slepton masses $(0.52$ and 0.04 versus 1.0) the correlation is much tighter for squarks than for sleptons, particularly for the left-handed stop. Furthermore, one sees that the slope of the correlation is fixed by the coefficients of $m_{1 / 2}$, which are much larger for squarks than for sleptons.

We note that, for any given value of $m_{1 / 2}$, the allowed ranges of $m_{\tilde{L}_{3}}$ and $m_{\tilde{E}_{3}}$ at the weak scale are lower than those of $m_{\tilde{Q}_{3}}$ and $m_{\tilde{U}_{3}}$, reflecting the greater renormalizations of the squark mass parameters, as seen by comparing the coefficients of $m_{1 / 2}$ in Eqs. (4) and (5). While several aspects of the MSSM spectrum enter in the calculation of the relic LSP density, the $\chi-p$ elastic scattering cross section is largely controlled by squark masses. We see in Figs. 1(a) and 1(b) that the slepton masses are much smaller than the squark masses shown in Figs. 1(c) and 1(d), implying that the $\chi$ $-p$ elastic scattering rate must be much smaller than would have been the case if the slepton and squark masses were comparable. Assuming small squark masses but large $m_{1 / 2}$, as might be done within the LEEST approach to yield relatively large cross sections for large LSP masses, would lead to the squarks becoming tachyonic long before the GUT scale. For another discussion of "perverse" models with tachyonic squarks at the GUT scale, see [25].

We further note that if we reduce the input scale, the correlations discussed above persist, but are somewhat weaker than in Fig. 1, where we had assumed Eq. (3) at the GUT scale.

Using these representative examples as a guide, we conclude, that even if one abandons the unification of the soft supersymmetry-breaking sfermion and Higgs-boson masses at the GUT scale, RGE evolution yields interesting correlations among the various parameters of the model also down at the weak scale. The fact that certain combinations of sfer- 

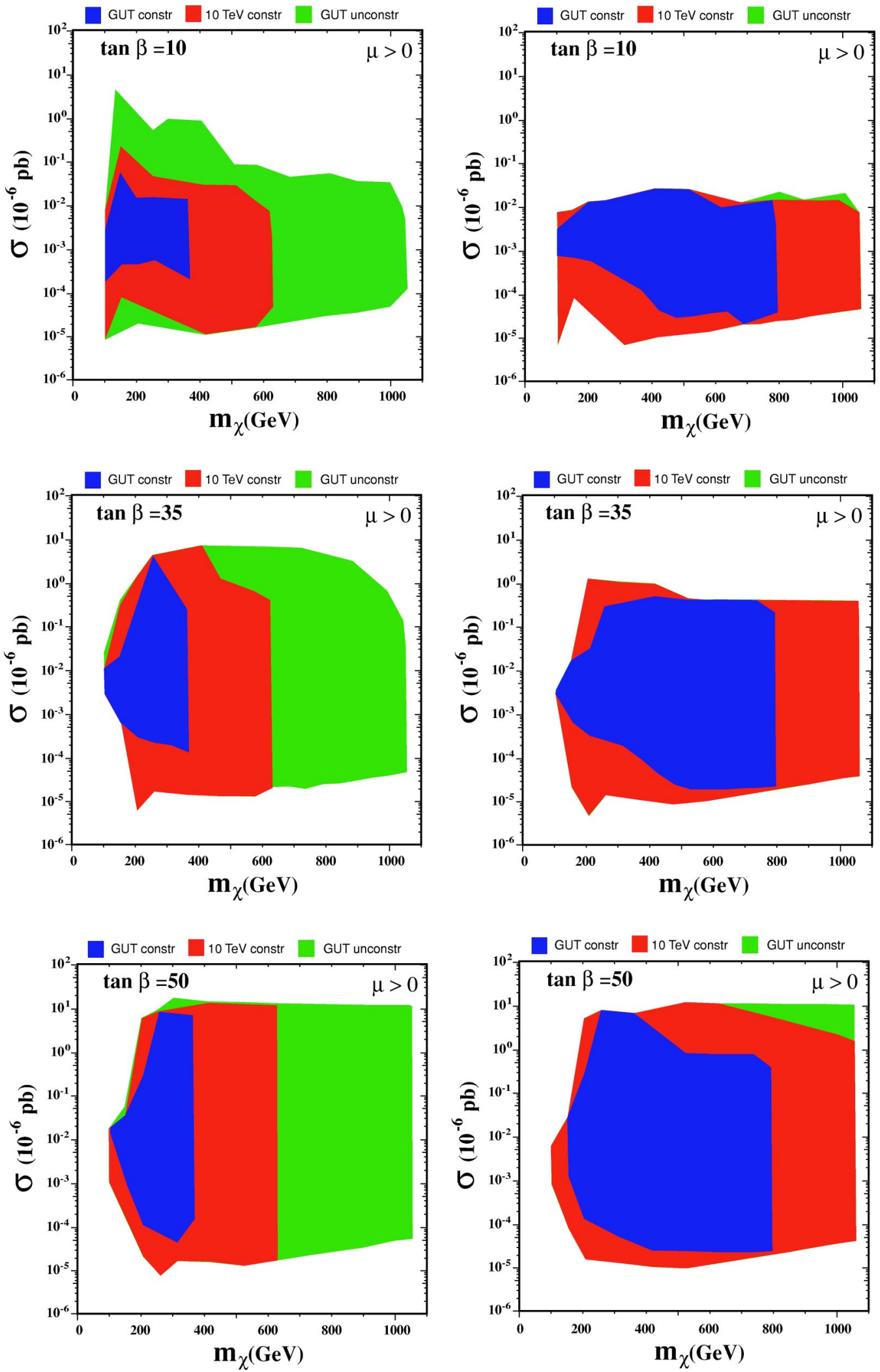

FIG. 2. The spin-independent part of the $\chi-p$ cross section for $\tan \beta=10,35$ and 50 in the top, middle, and bottom rows, respectively. The left (right) panels are for $200 \mathrm{GeV} \leqslant m_{\tilde{q}} \leqslant 2 \mathrm{TeV}\left(2 \mathrm{TeV} \leqslant m_{\tilde{q}} \leqslant 4 \mathrm{TeV}\right)$. The smallest (dark) and medium shaded regions are obtained requiring no tachyons before the GUT and $10 \mathrm{TeV}$ scales, respectively. The largest (light) shaded regions are obtained without any such constraint, and correspond to a general LEEST analysis. 

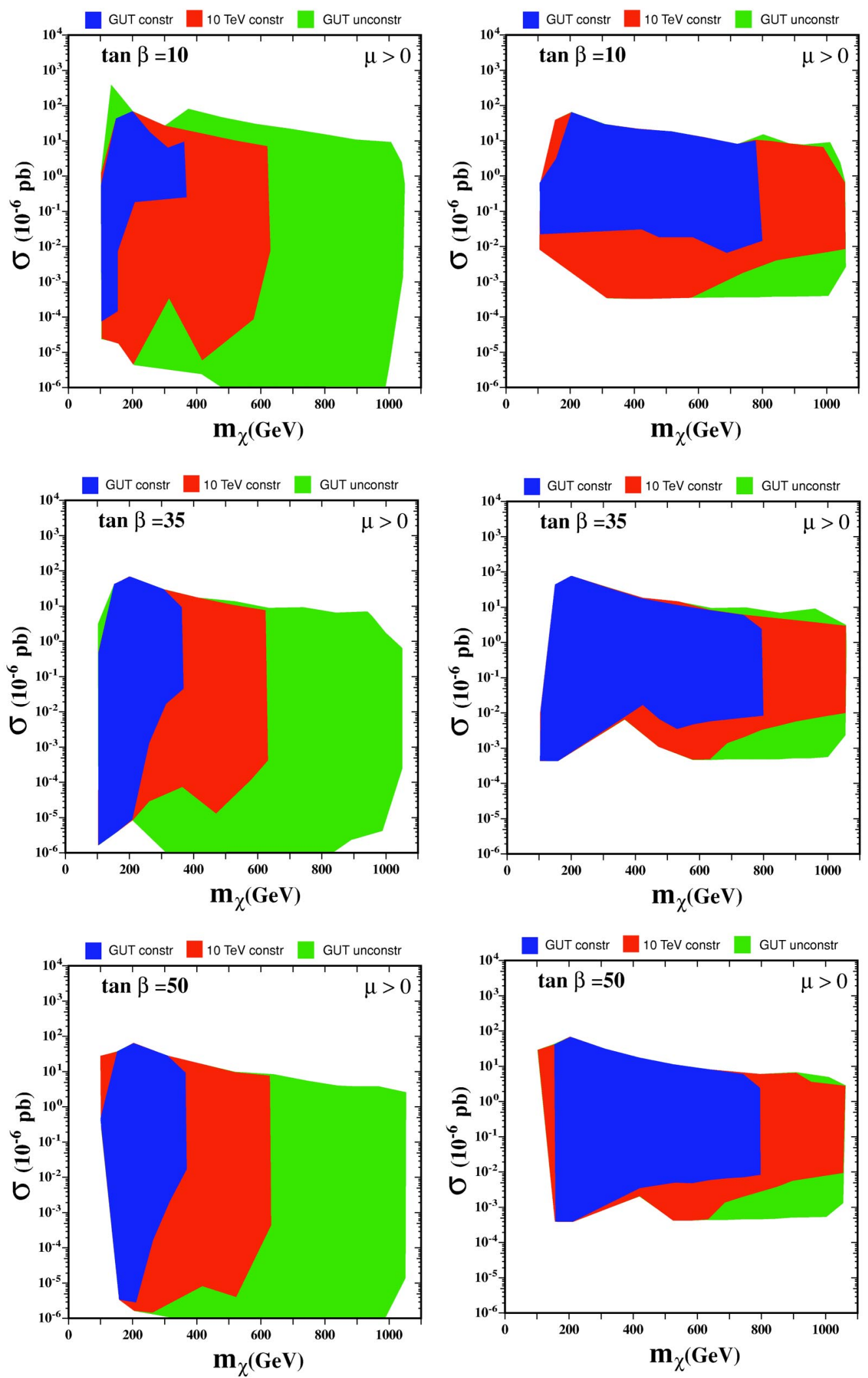

FIG. 3. The spin-dependent part of the $\chi-p$ cross section for $\tan \beta=10,35$, and 50 in the top, middle and bottom rows, respectively. The various regions are as in Fig. 2. 
mion masses and gaugino masses at the weak scale are never realized in a top-down analysis reflects the tachyonic constraint that we will apply in the following section. In particular, as already emphasized, if the physical squark and slepton masses are small and (nearly) equal, then, except possibly for small values of $m_{1 / 2}$, the squarks would become tachyonic at some scale not far above the weak scale. ${ }^{2}$

\section{LOW-ENERGY EFFECTIVE SUPERSYMMETRIC THEORY}

For our low-energy effective supersymmetric theory (LEEST) analysis, we use as our input parameters the SU(2) gaugino mass $M_{2}$, the Higgs mixing parameter $\mu$, the (assumed to be) universal soft supersymmetry-breaking slepton mass $m_{\tilde{\ell}}$, the universal squark mass $m_{\tilde{q}}$, the trilinear couplings $A_{t}, A_{b}$, and $A_{\tau}$, the pseudoscalar Higgs-boson mass $m_{A}$, and $\tan \beta$. We consider the following ranges for these parameters at the weak scale:

$$
\begin{gathered}
100 \mathrm{GeV} \leqslant M_{2} \leqslant 2 \mathrm{TeV}, \\
100 \mathrm{GeV} \leqslant m_{\tilde{\ell}} \leqslant 2 \mathrm{TeV}, \\
200 \mathrm{GeV} \leqslant m_{\tilde{q}} \leqslant 4 \mathrm{TeV}, \\
90 \mathrm{GeV} \leqslant m_{A} \leqslant 2 \mathrm{TeV}, \\
100 \mathrm{GeV} \leqslant|\mu| \leqslant 2 \mathrm{TeV} .
\end{gathered}
$$

We analyze in this paper only the $\mu>0$ case, fix $A_{t}=A_{b}$ $=1 \mathrm{TeV}$, assume $A_{\tau}=0$, and sample $\tan \beta=10,35$, and 50 . These assumptions have been chosen to resemble those of [18]. We note that other LEEST analyses [16] are more flexible, allowing $m_{\tilde{\ell}} \simeq m_{\tilde{q}}$ with both small (i.e., $<200 \mathrm{GeV}$ ), a possibility not included in Eq. (6). However, with regard to the discussion in the previous two sections, inclusion of the lower squark masses will almost certainly lead to tachyonic instabilities making the choice (6) somewhat better motivated. As usual, to obtain the physical sfermion masses, we add $D$-term corrections and diagonalize the mass matrices. The other gaugino masses, $M_{1}$ and $M_{3}$, are approximated by $M_{1}=\frac{5}{3} M_{2} \tan ^{2} \theta_{W}$ and $M_{3}=29.74 M_{2} \alpha_{s}$, so as to mimic gaugino unification at the GUT scale.

We should emphasize that this is an oversimplified model. First of all, there is no known fundamental principle why all the slepton and all the squark soft supersymmetry-breaking masses should each be universal at the weak scale. ${ }^{3}$ Moreover, physical masses should be calculated at their appropri-

\footnotetext{
${ }^{2}$ This statement depends on our assumption of universal gaugino masses at the GUT scale. If one assumed nonuniversal gaugino masses $M_{1,2,3}$, one would need $M_{3} \ll M_{1,2}$ to get nearly equal squark and slepton physical masses. This situation might not be suitable for providing dark matter.

${ }^{3}$ On the other hand, the experimental upper limits on flavorchanging neutral interactions suggest that the physical masses of particles with the same internal quantum numbers in different generations should be relatively degenerate [26].
}

ate scale, and sfermion masses at $M_{Z}$ could be significantly different from those at $M_{\text {susy }}$. Nevertheless, this toy model is often used in the literature $[16,17,18]$, and may be sufficient to represent a general analysis of the effective MSSM. It should be noted that, in this kind of analysis, one must make some simplifying assumptions, because otherwise the number of parameters is impractically large.

We apply the constraints on new particles from direct LEP searches, namely $m_{\chi^{ \pm}}>104 \mathrm{GeV}$ [27]; $m_{\tilde{e}}>99 \mathrm{GeV}$ [28], and Higgs mass limits [29]. We require the branching ratio for $b \rightarrow s \gamma$ to be consistent with the experimental measurements [30]. We do not impose the constraint suggested by $g_{\mu}-2$ [31], as the magnitude of the standard model contribution is still undetermined, in view of the discrepancy between the $e^{+} e^{-}$data and the $\tau$-decay data [32]. For the relic density of neutralinos $\chi$, we require that $0.1 \leqslant \Omega_{\chi} h^{2} \leqslant 0.3$. We use this more conservative range rather than that suggested by the recent WMAP data [24] $\left(0.094 \leqslant \Omega_{\chi} h^{2}\right.$ $\leqslant 0.129$ ), because it is more suitable for comparison with previous LEEST papers. In fact, we have performed the analogous analysis and, within the statistical errors of our samples, we have found that restricting MSSM so that the relic density falls within the WMAP range does not reduce significantly the ranges of the cross sections possible for any given value of $m_{\chi}$, and neither is the range of $m_{\chi}$ much different. This serves to emphasize the robustness of our results. For studies on the effect of the new WMAP density in CMSSM models, see [12].

We emphasize the importance of implementing correctly the available experimental constraints. For example, the SM limit of $m_{h}>114 \mathrm{GeV}$ [29] applies in the CMSSM, and also in the LEEST framework discussed here when $m_{A}$ is large. For small values of $m_{A}$ for which the ZZh coupling factor $\sin (\beta-\alpha)$ is less than a half, we relax the Higgs mass bound to $87 \mathrm{GeV}$. One might obtain much larger elastic scattering cross sections if one used a smaller lower limit on $m_{h}$. Similarly, the $b \rightarrow s \gamma$ constraint must be implemented taking careful account of the theoretical as well as the experimental errors, since it provides important lower limits on sparticle masses, in particular for $\mu<0$ and high values of $\tan \beta$.

\section{ELASTIC NEUTRALINO-PROTON SCATTERING CROSS SECTIONS}

Finally, we are ready to compute the elastic $\chi-p$ scattering cross section in the LEEST framework, using a scan in $100 \mathrm{GeV}$ steps of the parameter space over the ranges given in Eq. (6). For the points that pass all the phenomenological constraints mentioned earlier, we calculate the neutralinoproton cross section $\sigma_{\chi p}$ using the procedure described in [33]. We begin with the spin-independent (scalar) part of the cross section, and then turn to the spin-dependent part.

We discuss the spin-independent neutralino-proton cross section first in the general LEEST case, i.e., with parameters chosen in the low-energy effective MSSM and ignoring the effects of RGE running. The results are shown as the most extensive and lightest shaded regions in Fig. 2. There are six panels, two each for $\tan \beta=10,35,50$, in the top, middle and bottom rows of panels in the figure. The left panels have 

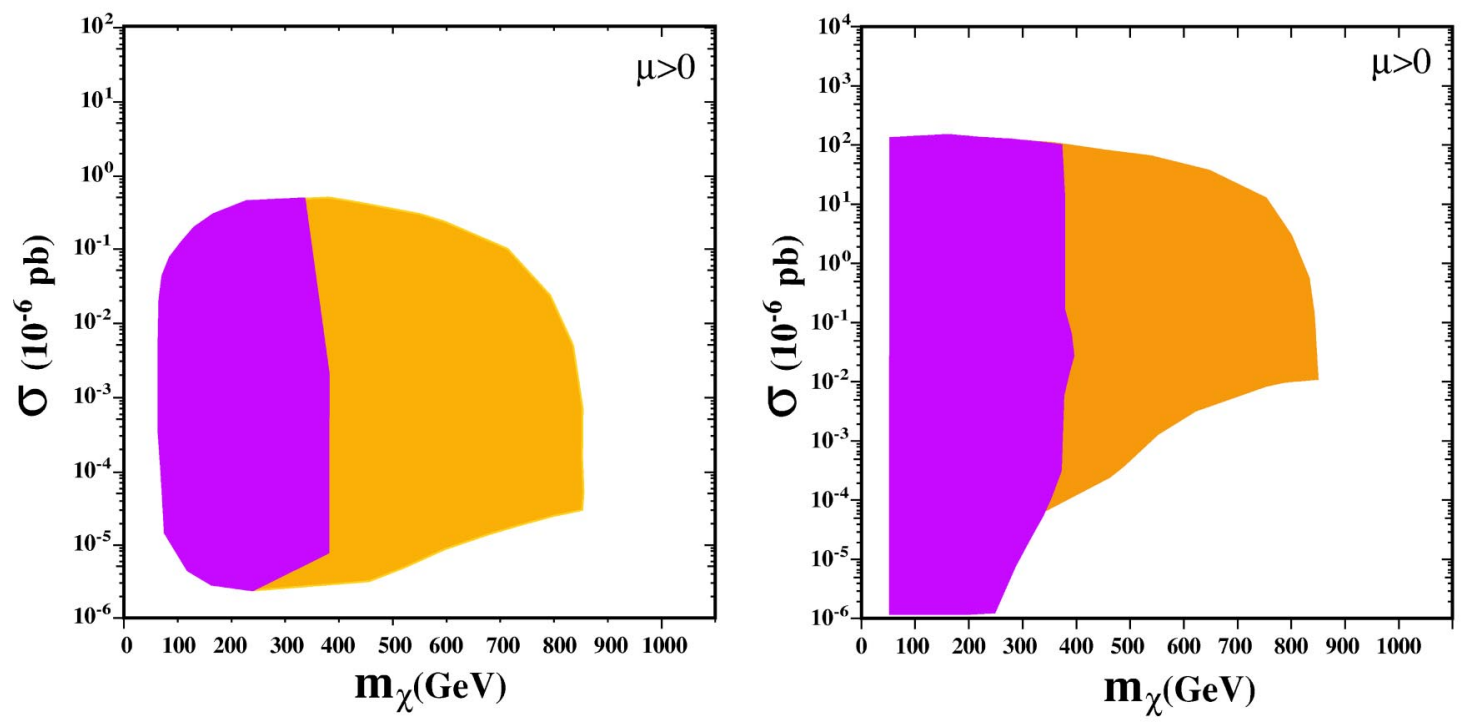

FIG. 4. Left (right) panels: the ranges of the spin-independent (spin-dependent) $\chi-p$ cross sections found in a top-down approach, requiring that there is no tachyon at any scale from $M_{\mathrm{GUT}}$ to $M_{Z}$ and combining all values of $\tan \beta$. The dark region corresponds to the low-mass range of squark masses $200 \mathrm{GeV}<m_{\tilde{q}}<2 \mathrm{TeV}$, while the light to the high $2 \mathrm{TeV}<m_{\tilde{q}}<4 \mathrm{TeV}$.

$200 \mathrm{GeV} \leqslant m_{\tilde{q}} \leqslant 2 \mathrm{TeV}$ and the right panels have $2 \mathrm{TeV}$ $\leqslant m_{\tilde{q}} \leqslant 4 \mathrm{TeV}$. We see that the maximum values of the cross section are higher in the left panels than in the right panels. The maximum cross section is usually dominated by the heavy Higgs exchange channel when the Higgsino content of the neutralino is large and $m_{A}$ is small. In this case, the charged Higgs mass is also small and as a consequence, the points with large scattering cross sections are excluded by the $b \rightarrow s \gamma$ constraint unless the squark masses are also relatively light. However, when the squarks are light, the RGE constraint is significant and points with large cross sections are excluded. We also notice that larger values of $\tan \beta$ have higher maximum cross sections, which is also known to be true for the CMSSM case. These results are in reasonably close agreement with those of [18].

When we evolve the MSSM RGEs up and demand that tachyonic sfermions be absent at the GUT scale $\sim 10^{16} \mathrm{GeV}$, we obtain the results shown as the smallest and darkest shaded regions in Fig. 2. In the left panels, these shaded regions are cut at $m_{\chi} \cong 400 \mathrm{GeV}$, whilst in the right panels they extend to $m_{\chi} \cong 800 \mathrm{GeV}$. This is due to the fact that, as we evolve to higher energies using the RGEs, the squark masses get smaller at rates that depend on the gaugino masses, as described in Sec. III. The larger the gaugino masses, the smaller the squark masses are at the GUT scale. As can be seen in Fig. 1, even if we start with $m_{\tilde{q}}\left(m_{Z}\right)$ $=2000 \mathrm{GeV}$, we find zero squark masses at the GUT scale for $m_{1 / 2}$ around $1000 \mathrm{GeV}$, corresponding to a neutralino mass around $400 \mathrm{GeV}$. If we increase the squark mass to $4000 \mathrm{GeV}, m_{1 / 2}$ can go up to around $2000 \mathrm{GeV}$, corresponding to $m_{\chi} \sim 800 \mathrm{GeV}$.

In the left panels of Fig. 2, we observe that the maximum cross section is generally lower than what would obtain if one did not impose the GUT constraint. This reflects the fact that, for any given value of $m_{\chi}$ and hence $m_{1 / 2}$, a lower limit on the low-energy effective squark masses is imposed by requiring the absence of tachyonic sfermions at the GUT scale. While this effect is quite pronounced at $\tan \beta=10$, we note that the reduction in the maximum cross section (for fixed $m_{\chi}$ ) is smaller at higher $\tan \beta$. Furthermore, at low values of $m_{\chi}(\sim 250 \mathrm{GeV})$, there is almost no reduction at all. In the panels on the right, no reduction is seen, as the squark masses are already so high that the RGE constraint is not effective.

Even if one does not require the absence of tachyons up to the GUT scale, their absence up to a lower effective scale still imposes important constraints on the MSSM, as is also seen in Fig. 2. The medium-sized and -shaded regions show the ranges of the spin-independent $\chi-p$ elastic cross section allowed if one forbids the existence of tachyons at scales below $10 \mathrm{TeV}$ rather than the GUT scale. We see that, even with this much-reduced maximal scale, the RGEs provide significant restrictions. In fact, most of the power of the nontachyonic constraint appears well below the GUT scale.

In Fig. 3 we show analogous results for the spindependent part of the $\chi-p$ elastic scattering cross section. Looking at the left or the right panels only, we see that the maximum cross sections are not greatly reduced by using the RGEs and requiring the absence of tachyons up to high energies. However, if we combine the left panels with the right ones, we nevertheless find that a smaller range of cross sections is allowed when we apply the RGE constraint than would be permitted without the RGE constraint, especially for large $m_{\chi}$.

Finally, we compare the above results with the range we find for the $\chi-p$ cross section if we start from the GUT scale with the ranges of input parameters described in Sec. II, and run down from $M_{\mathrm{GUT}}$ to $M_{Z}$. This top-down approach gives us Fig. 4, where all values of $\tan \beta$ are combined. To facilitate the comparison with the previous figures, we distinguish between the regions which give rise to squark masses above and below $2 \mathrm{TeV}$, corresponding to the right 
and left panels of the previous two figures. The area shown here should be compared to the dark shaded region corresponding to the GUT constraint. Indeed, the resulting ranges are similar to those found on the left side (both sides) of Fig. 2 , when one imposes the GUT constraint and combines all the three values of $\tan \beta$. Qualitatively, therefore, we find that the LEEST approach gives similar results to the GUT (top-down) approach, so long as the tachyonic stability constraints are imposed. We note, however, that the allowed cross-section ranges do differ from those in the CMSSM $[7,33,34]$, because the allowed parameter choices are no longer restricted to an extreme part of the coannihilation tail and, even within this tail, the other weak-scale sparticle masses have wider ranges than in the CMSSM. On the other hand, these results are very similar to the NUHM, which is a relaxed version of the CMSSM in which the Higgs soft masses are non-universal but the squark and slepton masses are universal [35].

\section{CONCLUSIONS}

We have compared in this paper predictions for neutralino-proton scattering in models that are free from ta- chyonic instabilities all the way up to the GUT scale, or just up to $10 \mathrm{TeV}$, with those of models in which only a lowenergy effective supersymmetric theory (LEEST) is assumed, and no restriction is placed on its behavior above the weak scale. As we have shown, larger LSP masses and larger cross sections are obtainable in the LEEST approach, but at the risk of sacrificing some of the major motivations for postulating low-energy supersymmetry. Specifically, we argue that a generic LEEST cannot be extrapolated up to the GUT scale. The main issue is whether the tachyonic instabilities that are generic in the LEEST approach are tolerable. Clearly in any theory with GUT scale unification, they are not. Cross sections larger than those in the CMSSM may be attainable, particularly at large LSP masses, if such tachyonic instabilities are allowed.

\section{ACKNOWLEDGMENTS}

We would like to thank L. Roszkowski, M. Shifman, and M. Srednicki for helpful comments. The work of K.A.O., Y.S., and V.C.S. was supported in part by DOE Grant No. DE-FG02-94ER-40823.
[1] E. Witten, Nucl. Phys. B188, 513 (1981); N. Sakai, Z. Phys. C 11, 153 (1981); S. Dimopoulos and H. Georgi, Nucl. Phys. B193, 150 (1981); R. K. Kaul and P. Majumdar, ibid. B199, 36 (1982).

[2] J. R. Ellis, S. Kelley, and D. V. Nanopoulos, Phys. Lett. B 260, 131 (1991); U. Amaldi, W. de Boer, and H. Furstenau, ibid. 260, 447 (1991); P. Langacker and M. Luo, Phys. Rev. D 44, 817 (1991); C. Giunti, C. W. Kim, and U. W. Lee, Mod. Phys. Lett. A 6, 1745 (1991).

[3] J. Ellis, J. S. Hagelin, D. V. Nanopoulos, K. A. Olive, and M. Srednicki, Nucl. Phys. B238, 453 (1984); see also H. Goldberg, Phys. Rev. Lett. 50, 1419 (1983).

[4] For a review see, e.g., H. E. Haber and G. L. Kane, Phys. Rep. 117, 75 (1985).

[5] L. Girardello and M. T. Grisaru, Nucl. Phys. B194, 65 (1982).

[6] M. Drees and M. M. Nojiri, Phys. Rev. D 47, 376 (1993); H. Baer and M. Brhlik, ibid. 53, 597 (1996); J. R. Ellis, T. Falk, K. A. Olive, and M. Schmitt, Phys. Lett. B 388, 97 (1996); 413, 355 (1997); J. R. Ellis, T. Falk, G. Ganis, K. A. Olive, and M. Schmitt, Phys. Rev. D 58, 095002 (1998); J. R. Ellis, T. Falk, G. Ganis, and K. A. Olive, ibid. 62, 075010 (2000); J. R. Ellis, T. Falk, G. Ganis, K. A. Olive, and M. Srednicki, Phys. Lett. B 510, 236 (2001); V. D. Barger and C. Kao, Phys. Rev. D 57, 3131 (1998); J. L. Feng, K. T. Matchev, and T. Moroi, Phys. Rev. Lett. 84, 2322 (2000); Phys. Rev. D 61, 075005 (2000); A. B. Lahanas, D. V. Nanopoulos, and V. C. Spanos, ibid. 62, 023515 (2000).

[7] H. Baer and M. Brhlik, Phys. Rev. D 57, 567 (1998); J. L. Feng, K. T. Matchev, and F. Wilczek, Phys. Lett. B 482, 388 (2000)

[8] J. R. Ellis, T. Falk, and K. A. Olive, Phys. Lett. B 444, 367 (1998); J. R. Ellis, T. Falk, K. A. Olive, and M. Srednicki,
Astropart. Phys. 13, 181 (2000); 15, 413(E) (2001).

[9] M. E. Gómez, G. Lazarides, and C. Pallis, Phys. Rev. D 61, 123512 (2000); Phys. Lett. B 487, 313 (2000); Nucl. Phys. B638, 165 (2002); R. Arnowitt, B. Dutta, and Y. Santoso, ibid. B606, 59 (2001); T. Nihei, L. Roszkowski, and R. Ruiz de Austri, J. High Energy Phys. 07, 024 (2002); H. Baer, C. Balazs, and A. Belyaev, ibid. 03, 042 (2002); G. Belanger, F. Boudjema, A. Pukhov, and A. Semenov, Comput. Phys. Commun. 149, 103 (2002); J. Edsjo, M. Schelke, P. Ullio, and P. Gondolo, J. Cosmol. Astropart. Phys. 04, 001 (2003).

[10] C. Boehm, A. Djouadi, and M. Drees, Phys. Rev. D 62, 035012 (2000); J. R. Ellis, K. A. Olive, and Y. Santoso, Astropart. Phys. 18, 395 (2003).

[11] V. D. Barger and C. Kao, Phys. Lett. B 518, 117 (2001); L. Roszkowski, R. Ruiz de Austri, and T. Nihei, J. High Energy Phys. 08, 024 (2001); A. B. Lahanas and V. C. Spanos, Eur. Phys. J. C 23, 185 (2002); A. Djouadi, M. Drees, and J. L. Kneur, J. High Energy Phys. 08, 055 (2001); U. Chattopadhyay, A. Corsetti, and P. Nath, Phys. Rev. D 66, 035003 (2002); J. R. Ellis, K. A. Olive, and Y. Santoso, New J. Phys. 4, 32 (2002); H. Baer, C. Balazs, A. Belyaev, J. K. Mizukoshi, X. Tata, and Y. Wang, J. High Energy Phys. 07, 050 (2002); R. Arnowitt and B. Dutta, hep-ph/0211417.

[12] J. R. Ellis, K. A. Olive, Y. Santoso, and V. C. Spanos, Phys. Lett. B 565, 176 (2003); H. Baer and C. Balazs, J. Cosmol. Astropart. Phys. 05, 006 (2003); A. B. Lahanas and D. V. Nanopoulos, Phys. Lett. B 568, 55 (2003); U. Chattopadhyay, A. Corsetti, and P. Nath, Phys. Rev. D 68, 035005 (2003).

[13] M. Drees, M. M. Nojiri, D. P. Roy, and Y. Yamada, Phys. Rev. D 56, 276 (1997); 64, 039901(E) (2001); M. Drees, Y. G. Kim, M. M. Nojiri, D. Toya, K. Hasuko, and T. Kobayashi, ibid. 63, 035008 (2001); V. Berezinsky, A. Bottino, J. R. Ellis, N. 
Fornengo, G. Mignola, and S. Scopel, Astropart. Phys. 5, 1 (1996); P. Nath and R. Arnowitt, Phys. Rev. D 56, 2820 (1997); A. Bottino, F. Donato, N. Fornengo, and S. Scopel, ibid. 63, 125003 (2001); S. Profumo, ibid. 68, 015006 (2003).

[14] J. Ellis, K. Olive, and Y. Santoso, Phys. Lett. B 539, 107 (2002); J. R. Ellis, T. Falk, K. A. Olive, and Y. Santoso, Nucl. Phys. B652, 259 (2003).

[15] K. Griest and L. Roszkowski, Phys. Rev. D 46, 3309 (1992); S. Mizuta and M. Yamaguchi, Phys. Lett. B 298, 120 (1993); S. Mizuta, D. Ng, and M. Yamaguchi, ibid. 300, 96 (1993); J. Edsjo and P. Gondolo, Phys. Rev. D 56, 1879 (1997); V. Bertin, E. Nezri, and J. Orloff, J. High Energy Phys. 02, 046 (2003); A. Birkedal-Hansen and E. h. Jeong, ibid. 02, 047 (2003); A. Birkedal-Hansen and B. D. Nelson, Phys. Rev. D 67, 095006 (2003); U. Chattopadhyay and D. P. Roy, ibid. 68, 033010 (2003).

[16] L. Bergstrom and P. Gondolo, Astropart. Phys. 5, 263 (1996); V. A. Bednyakov and H. V. Klapdor-Kleingrothaus, Phys. Rev. D 62, 043524 (2000); V. Mandic, A. Pierce, P. Gondolo, and H. Murayama, hep-ph/0008022; A. Bottino, V. de Alfaro, N. Fornengo, G. Mignola, and M. Pignone, Astropart. Phys. 2, 67 (1994); A. Bottino, F. Donato, N. Fornengo, and S. Scopel, Phys. Rev. D 59, 095003 (1999); 59, 095004 (1999); 62, 056006 (2000); 63, 125003 (2001); hep-ph/0307303.

[17] K. A. Olive and M. Srednicki, Phys. Lett. B 230, 78 (1989); Nucl. Phys. B355, 208 (1991); K. Griest, M. Kamionkowski, and M. S. Turner, Phys. Rev. D 41, 3565 (1990); A. Bottino, G. Mignola, M. Olechowski, and S. Scopel, Phys. Lett. B 380, 308 (1996); V. A. Bednyakov, H. V. Klapdor-Kleingrothaus, and E. Zaiti, Phys. Rev. D 66, 015010 (2002); V. A. Bednyakov, H. V. Klapdor-Kleingrothaus, and V. Gronewold, ibid. 66, 115005 (2002); E. A. Baltz and P. Gondolo, Phys. Rev. Lett. 86, 5004 (2001); Phys. Rev. D 67, 063503 (2003).

[18] Y. G. Kim, T. Nihei, L. Roszkowski, and R. Ruiz de Austri, J. High Energy Phys. 12, 034 (2002).

[19] T. Falk, K. A. Olive, L. Roszkowski, and M. Srednicki, Phys. Lett. B 367, 183 (1996).

[20] J. A. Casas, A. Lleyda, and C. Munoz, Nucl. Phys. B471, 3 (1996); Phys. Lett. B 389, 305 (1996); A. Strumia, Nucl. Phys. B482, 24 (1996); H. Baer, M. Brhlik, and D. Castano, Phys. Rev. D 54, 6944 (1996); S. A. Abel and C. A. Savoy, Nucl. Phys. B532, 3 (1998); S. Abel and T. Falk, Phys. Lett. B 444, 427 (1998); D. G. Cerdeno, E. Gabrielli, M. E. Gomez, and C. Munoz, J. High Energy Phys. 06, 030 (2003).

[21] A. Riotto and E. Roulet, Phys. Lett. B 377, 60 (1996).

[22] A. Kusenko, P. Langacker, and G. Segre, Phys. Rev. D 54, 5824 (1996).

[23] T. Falk, K. A. Olive, L. Roszkowski, A. Singh, and M. Srednicki, Phys. Lett. B 396, 50 (1997).

[24] C. L. Bennett et al., Astrophys. J., Suppl. Ser. 148, 1 (2003); D. N. Spergel et al., ibid. 148, 175 (2003).

[25] G. L. Kane, B. D. Nelson, T. T. Wang, and L. T. Wang, hep-ph/0304134.

[26] J. R. Ellis and D. V. Nanopoulos, Phys. Lett. 110B, 44 (1982).

[27] Joint LEP 2 Supersymmetry Working Group, Combined LEP
Chargino Results, up to $208 \mathrm{GeV}$, http://lepsusy.web.cern.ch/ lepsusy/www/inos_moriond01/charginos_pub.html

[28] Joint LEP 2 Supersymmetry Working Group, Combined LEP Selectron/Smuon/Stau Results, 183-208 GeV, http:// lepsusy.web.cern.ch/lepsusy/www/sleptons_summer02/ slep_2002.html

[29] LEP Higgs Working Group for Higgs boson searches, OPAL Collaboration, ALEPH Collaboration, DELPHI Collaboration, and L3 Collaboration, Phys. Lett. B 565, 61 (2003). Searches for the neutral Higgs bosons of the MSSM: Preliminary combined results using LEP data collected at energies up to 209 GeV, LHWG-NOTE-2001-04, ALEPH-2001-057, DELPHI2001-114, L3-NOTE-2700, OPAL-TN-699, hep-ex/0107030; LHWG Note/2002-01, http://lephiggs.web.cern.ch/ LEPHIGGS/papers/July2002_SM/index.html

[30] CLEO Collaboration, M. S. Alam et al., Phys. Rev. Lett. 74, 2885 (1995); as updated in S. Ahmed et al., CLEO CONF 99-10; Belle Collaboration, contribution to the 30th International conference on High-Energy Physics, Osaka, 2000, BELLE-CONF-0003; see also Belle Collaboration, K. Abe et al., hep-ex/0107065; BaBar Collaboration, L. Lista, hep-ex/0110010; C. Degrassi, P. Gambino, and G. F. Giudice, J. High Energy Phys. 12, 009 (2000); M. Carena, D. Garcia, U. Nierste, and C. E. Wagner, Phys. Lett. B 499, 141 (2001); D. A. Demir and K. A. Olive, Phys. Rev. D 65, 034007 (2002); T. Hurth, hep-ph/0106050.

[31] Muon g-2 Collaboration, G. W. Bennett et al., Phys. Rev. Lett. 89, 101804 (2002); 89, 129903(E) (2002).

[32] M. Davier, S. Eidelman, A. Hocker, and Z. Zhang, Eur. Phys. J. C 27, 497 (2003); see also K. Hagiwara, A. D. Martin, D. Nomura, and T. Teubner, Phys. Lett. B 557, 69 (2003); F. Jegerlehner (unpublished); as reported in M. Krawczyk, Acta Phys. Pol. B 33, 2621 (2002).

[33] J. R. Ellis, A. Ferstl, and K. A. Olive, Phys. Lett. B 481, 304 (2000); Phys. Rev. D 63, 065016 (2001); Phys. Lett. B 532, 318 (2002).

[34] K. Griest, Phys. Rev. D 38, 2357 (1988); J. Ellis and R. Flores, Nucl. Phys. B307, 883 (1988); R. Barbieri, M. Frigeni, and G. Giudice, ibid. B313, 725 (1989); R. Flores, K. A. Olive, and M. Srednicki, Phys. Lett. B 237, 72 (1990); J. Ellis and R. Flores, ibid. 263, 259 (1991); 300, 175 (1993); M. Drees and M. Nojiri, Phys. Rev. D 48, 3483 (1993); V. Bednyakov, H. V. Klapdor-Kleingrothaus, and S. Kovalenko, ibid. 50, 7128 (1994); R. Arnowitt and P. Nath, ibid. 54, 2374 (1996); A. Corsetti and P. Nath, ibid. 64, 125010 (2001); E. Accomando, R. Arnowitt, B. Dutta, and Y. Santoso, Nucl. Phys. B585, 124 (2000); M. Drees, Y. G. Kim, T. Kobayashi, and M. M. Nojiri, Phys. Rev. D 63, 115009 (2001); M. E. Gómez and J. D. Vergados, Phys. Lett. B 512, 252 (2001); Y. G. Kim and M. M. Nojiri, Prog. Theor. Phys. 106, 561 (2001); A. B. Lahanas, D. V. Nanopoulos, and V. C. Spanos, Mod. Phys. Lett. A 16, 1229 (2001); Phys. Lett. B 518, 94 (2001); H. Baer, C. Balazs, A. Belyaev, and J. O'Farrill, J. Cosmol. Astropart. Phys. 09, 007 (2003).

[35] J. R. Ellis, A. Ferstl, K. A. Olive, and Y. Santoso, Phys. Rev. D 67, 123502 (2003). 